\title{
Merits and Limits: Applying open data to monitor open access publications in bibliometric databases
}

\author{
Aliakbar Akbaritabar* \\ Stephan Stahlschmidt
}

\begin{abstract}
Identifying and monitoring Open Access (OA) publications might seem a trivial task while practical efforts prove otherwise. Contradictory information arise often depending on metadata employed. We strive to assign OA status to publications in Web of Science (WOS) and Scopus while complementing it with different sources of OA information to resolve contradicting cases. We linked publications from WOS and Scopus via DOIs and ISSNs to Unpaywall, Crossref, DOAJ and ROAD. Only about 50\% of articles and reviews from WOS and Scopus could be matched via a DOI to Unpaywall. Matching with Crossref brought 56 distinct licences, which define in many cases the legally binding access status of publications. But only $44 \%$ of publications hold only a single licence on Crossref, while more than $50 \%$ have no licence information submitted to Crossref. Contrasting OA information from Crossref licences with Unpaywall we found contradictory cases overall amounting to more than $25 \%$, which might be partially explained by (ex-)including green OA. A further manual check found about $17 \%$ of OA publications that are not accessible and $15 \%$ non-OA publications that are accessible through publishers' websites. These preliminary results suggest that identification of OA state of publications denotes a difficult and currently unfulfilled task.
\end{abstract}

Keywords: Open Access, Unpaywall, Crossref, Web Of Science, Scopus

\section{Introduction}

Open access (henceforth OA) in scholarly communication describes unrestricted access to published peerreviewed documents written by and addressed to researchers. These documents have traditionally been disseminated via publications in scientific journals, which charge for access to the respective content. Stimulated by a call for greater openness and transparency in general ("open science"), the OA movement has nowadays been accepted as one, though not the only, alternative for the dissemination of scholarly documents. Even publishers seem to embrace this new model as providing a suitable infrastructure while at the same time securing their own economic interests.

This inter-mixture of interests has resulted not only in one, but several forms of OA publications such as Gold, Hidden Gold, Hybrid, Green, Delayed, Bronze and Black which are mainly based on right to access and pay to publish models depending on venues where the OA publication is accessible.

Due to the individual ascription of single publications to one or several of these categories and the decentralized structure of the scientific publishing market with a variety of diverse publishers, the identification of OA is less trivial than it might seem. Even large bibliometric data provider rely on external information to provide information on $\mathrm{OA}^{1}$ and most large scale undertakings by the scientometric community to obtain reliable information on OA prevalence rely on the use of web crawlers (Archambault et al., 2013; Piwowar et al., 2018)

Inspired by the Hybrid OA Dashboard (Jahn, 2017) we applied licensing information detailing the legally binding access state supplied by publishers to the publisher association Crossref to identify OA publications. We determined the OA status of all publications retrieved from Web of Science (henceforth WOS) and Scopus in-house databases of 2017 by confronting them to two sources of OA information, i.e., Unpaywall

\footnotetext{
${ }^{*}$ German Centre for Higher Education Research and Science Studies (DZHW), Schützenstr. 6a, Berlin, 10117 (Germany); akbaritabar@dzhw.eu; (corresponding author)

${ }^{\dagger}$ German Centre for Higher Education Research and Science Studies (DZHW); stahlschmidt@dzhw.eu

${ }^{1}$ https://clarivate.com/blog/easing-access-to-open-access-clarivate-analytics-partners-with-impactstory/
} 
and Crossref. In Section 2, we present our data and methods. In Section 3 we present our findings, while we discuss our main results in Section 4.

\section{Data \& Method}

We queried all publications from Scopus and WOS in in-house databases of 2017. Data included article's unique ID from database and DOI. We matched those DOIs with Unpaywall database from April $18^{\text {th }} 2018$ to determine the OA status for each single publication. In parallel, we matched those DOIs with Crossref data (using snapshot of the data from April 2018 based on plus service described in Crossref (2018)) and retrieved the available information on the licences of publications ${ }^{2}$.

Additionally, we used the journals' ISSNs provided by Wohlgemuth, Rimmert, \& Winterhager (2016) (and the updated version in Rimmert, Bruns, Lenke, \& Taubert (2017)) to identify Gold OA publications. They use different known OA indexes (e.g., DOAJ ${ }^{3}$ (Directory of Open Access Journals) and ROAD ${ }^{4}$ (Directory of Open Access scholarly Resources) and determine if the respective ISSN is listed in those databases. They differentiate between $I S S N$ and $I S S N L$ which is more fine-grained by adding a specific ISSN to some special issues. We tried both ISSN and ISSNL, sicne the latter had higher matching records, therefore in our analysis presented in the Results section we use the ISSNL.

It is necessary to note that some publications had multiple licence URLs in Crossref database, we followed a procedure with four steps to ensure using only one licence per publication (see Table 2 for the frequencies of these publications):

1. If a publication had only one record in Crossref database, whether it had an OA, non-OA, unclear licence or no licence information (i.e. $N A$ ), we used this status and categorized the publication as a unique one.

2. If a publication had multiple $O A$ licence URLs, we removed the duplicates and categorized it as $O A$.

3. If a publication had a mixture of $O A$ and non- $O A$ licence URLs, we removed the duplicates and categorized it as $O A$.

4. If a publication had multiple non-OA licence URLs, we removed the duplicates and categorized it as non-OA.

A research assistant controlled the unique licences (a total of 56) we extracted from Crossref with available information online to categorize them as $O A$ and non- $O A$. We used this categorization in parallel to established OA identification procedures (e.g., searching for journal's ISSN in DOAJ and ROAD in Gold OA identification) to ensure a higher level of robustness in our results.

In OA Identification process and in order to determine if a publication was OA or not, we applied a multi-category view separating Gold, Hidden Gold, Hybrid and Delayed OA, while doing so, we reached a new category of Probable Hybrid OA. Our investigation strategy for each category was as follows:

- Gold OA: As described earlier, we used the ISSNs provided by Rimmert et al. (2017) to determine Gold OA. We matched the respective ISSN (from both WOS and Scopus) with DOAJ and ROAD. If the respective ISSN was listed in one of those directories, the publication is categorized as Gold OA. We confronted Gold OA from DOAJ and ROAD with our research assistant's categorization of Crossref licences after the manual check of unique licence URLs.

- Hidden Gold OA: we used metadata from WOS and Scopus to determine the journal issue and looking at the licences of all publications in a single issue, if all publications had $O A$ licences, but the ISSN was not indexed in DOAJ or ROAD we categorized it as Hidden Gold OA.

\footnotetext{
${ }^{2}$ It is neccessary to note that our effort to send large number of requests to Crossref API (even while using plus service and through both rcrossref package in $\mathrm{R}$ and more fine-grained httr requests directly to Crossref API) faced timeout and response time errors and alternatively we chose to use the in-house snapshot of the Crossref data to circumvent the above error. This meant parsing large corpus of JSON files which can be time consuming depending on the goals of the analysis. Any effort on automating the proposed OA identification procedure needs to overcome the technical issues like this.

${ }^{3}$ https://doaj.org/

${ }^{4}$ https://road.issn.org/
} 
Table 1: All publications from WOS (2000-2017) and Scopus (2000-2017) that have an equivalent record in Unpaywall database (joined by DOIs)

\begin{tabular}{l|l|l}
\hline Data Source & Frequency & Percent \\
\hline WOS (matched Unpaywall Only articles \& reviews) & $11,661,206$ & $57.5 \%$ \\
\hline WOS (Only articles \& reviews 2000-2017) & $20,280,606$ & - \\
\hline Scopus (matched Unpaywall Only articles \& reviews) & $14,188,983$ & $53.48 \%$ \\
\hline Scopus (Only articles \& reviews 2000-2017) & $26,532,295$ & - \\
\hline
\end{tabular}

- Hybrid OA: If an issue had at least one non-OA publication while having one or more $O A$ publications, we categorized the OA publications as Hybrid OA.

- Probable Hybrid OA: If an issue did not have a non-OA publication while having one or more $O A$ publications and some publications in the issue didn't have licence information, we categorized them as Probable Hybrid OA.

- Delayed OA: In all of the above cases, we looked into delays based on Crossref metadata (a difference in terms of days from day of publication and the date licence was assigned to the publication as described in CrossRef-API (2019), this is the time period known as embargo time) to determine if they were Delayed, therefore each of the above categories were split to two groups, delayed and not-delayed. If a publication had multiple licence URLs on Crossref, we controlled their respective delay times, if any of those were not-delayed we categorized the publication as such, while if any of the licences were delayed, the publication is identified as a delayed one.

- Closed Access: Strictly speaking, if the number of publications in an issue was equal to the number of non-OA publications and the ISSN was not indexed in DOAJ or ROAD, we categorized them as Closed Access.

- NA (Not available): A publication that was not fitting in any of the above categories or did not have a licence URL to determine its condition was categorised as NA. Number of NAs are higher than Closed Access publications, since we aimed to keep the definitions as strict as possible.

\section{Results}

We present the results in two main sections, one regarding Unpaywall and the other on licences extracted from Crossref. We then present the comparison between Unpaywall and Crossref and the results of our manual checks on random samples for robustness of the results.

Table 1 shows the number of articles and review papers from WOS and Scopus with an equivalent record in Unpaywall database. It presents also the total number of articles and review papers in WOS/Scopus to provide a baseline for comparison. Unpaywall has higher than $50 \%$ coverage in both cases while coverage of WOS is slightly higher (can be due to different indexing philosophy or DOIs completeness). In the following tables (in Unpaywall results), publications are limited to only articles and review papers published in 2000-2017.

Figure 1 presents the distribution of journals and publications indexed in WOS (top) and Scopus (bottom) matched with Unpaywall database and crosschecked the ISSNs with DOAJ. Missing on DOAJ in these Figures refer to those journals whose ISSN was missing from Rimmert et al. (2017) data, therefore we could not check if the ISSN is listed in DOAJ or not while Others means the ISSN was existing in Rimmert et al. (2017) but it was not listed as $O A$ in DOAJ. Share of pubilcations which don't have a matching ISSN in DOAJ (meaning they are not Gold OA) and are identified as OA in Unpaywall is interesting on both Figures (designated with "Missing on DOAJ | Unpaywall OA" as label). They could be other OA types (green, hybrid, hidden gold).

We matched publications to Crossref data from April 2018 and found 56 distinct licence types for all of the publications. Table 2 presents a descriptive view on whether publications have licence information recorded in Crossref. It shows that about $50 \%$ of publications from WOS or Scopus with a matching DOI indexed in Crossref do not have a licence URL. Some of the publications had more than one licence information in Crossref (as an example, the number of DOIs that each have 6 licence records on Crossref are 7 ). In case of 

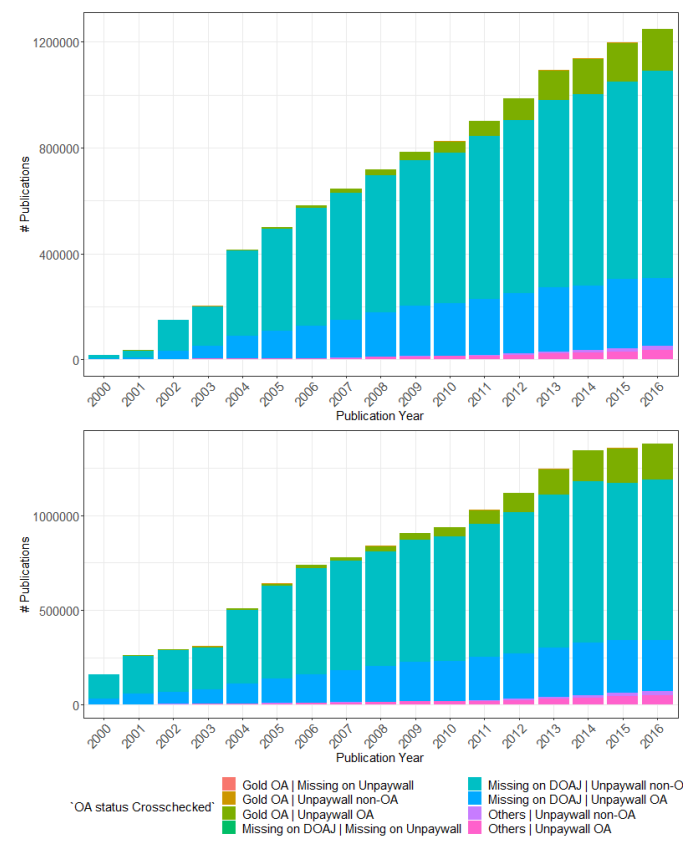

Figure 1: Publications indexed in WOS (top) and Scopus (bottom) matched with Unpaywall database and crosschecked the ISSNs with DOAJ (Gold OA) (X-axis denotes the years, Y-axis denotes the number of publications in each year)

Table 2: Number of licences per DOI found in Crossref

\begin{tabular}{r|r|r}
\hline Number of licences per DOI & Frequency of DOIs & Percent \\
\hline 0 & $9,892,208$ & 51.41 \\
\hline 1 & $8,520,158$ & 44.28 \\
\hline 2 & 824,975 & 4.29 \\
\hline 3 & 5,770 & 0.03 \\
\hline 5 & 25 & 0.00 \\
\hline 6 & 7 & 0.00 \\
\hline
\end{tabular}

multiple licences, if a publication had at least one OA licence, we categorized it as $O A$.

Figure 2 present the Gold, Hidden Gold, Hybrid and Delayed OA status of the publications from WOS (top) and Scopus (bottom), which is presented as trends over the years. We limited the years to 2000-2017 to show the most recent trends. To make these Figures more readable, we removed $N A$ (those without a matching DOI or without a licence information on Crossref).

Tables 3 and 4 present the OA status comparison between Unpaywall and Crossref in WOS and Scopus publications, respectively. Note, Crossref OA status in the Tables is the categorization we developed using respective licence URLs. We double checked the contradictory cases and improved our while-list of OA licences, while some of the contradictions still remain (e.g., Unpaywall declares those publications as OA while they are closed access or vice versa, in case of licences on Crossref that are open access while the publication is declared as non-OA on Unpaywall). Overall contradictory cases amount to $27.95 \%$ in WOS and $27.57 \%$ in Scopus which might partly be explained by the wider scope of Unpaywall including also green OA publications that might not be identified via license information only.

Tables 5 and 6 present the result of our research assistant's manual check for accessibility to article's PDF file from publishers websites compared to the respecitve licence in Crossref and the OA status we manually assignded to those URLs in contrast to OA status from Unpaywall. It is interesting to see there are publications defined as Non-OA while their PDF is accessible from the publisher (14.42\% in WOS and $14.98 \%$ in Scopus) or vice versa, OA publications (based on either Unpaywall, Crossref or both) that are not accessible online 

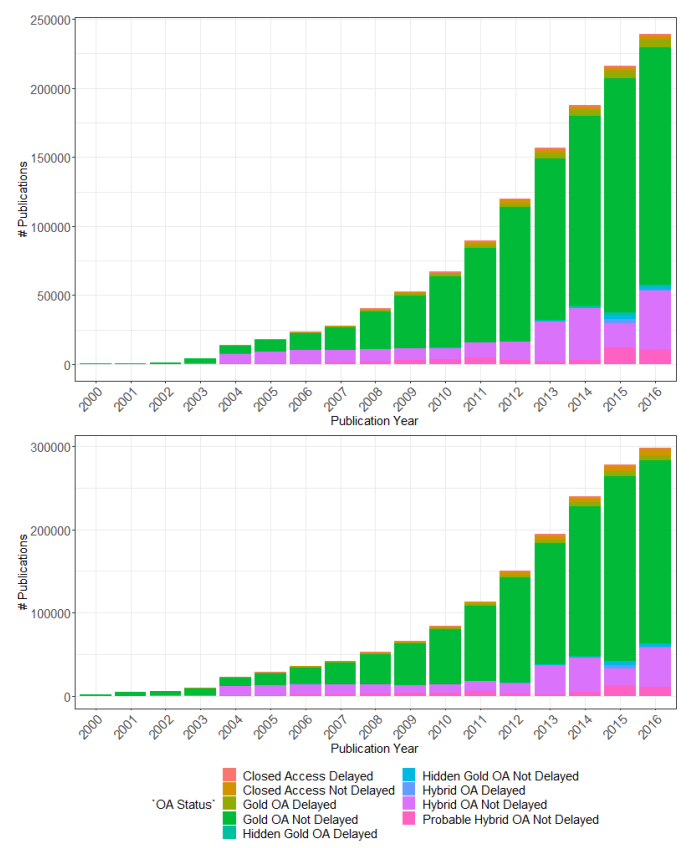

Figure 2: Comparison of OA publications 2000-2017 (WOS (top) and Scopus (bottom) data matched with Crossref) (X-axis denotes the years, $\mathrm{Y}$-axis denotes the number of publications in each year)

Table 3: OA status comparison between Unpaywall and Crossref in WOS publications

\begin{tabular}{l|l|r|r}
\hline Crossref OA Status & Unpaywall OA Status & Frequency & Percent \\
\hline Closed Access & Closed Access & $4,452,185$ & 38.18 \\
\hline NA & Closed Access & $3,512,794$ & 30.12 \\
\hline NA & Open Access & $1,770,612$ & 15.18 \\
\hline Closed Access & Open Access & $1,363,525$ & 11.69 \\
\hline Open Access & Open Access & 435,516 & 3.73 \\
\hline Open Access & Closed Access & 126,354 & 1.08 \\
\hline Closed Access & NA & 26 & 0.00 \\
\hline NA & NA & 19 & 0.00 \\
\hline
\end{tabular}

( $17.57 \%$ in WOS and $16.74 \%$ in Scopus). Note also the contradictory cases between Crossref and Unpaywall, where metadata from one shows $O A$ and the other Closed, which requires further probes $22.98 \%$ in WOS and $22.91 \%$ in Scopus, these percentages are quite close to contradictions observed in the overall sample presented in Tables 3 and 4). Our effort to complement these databases proves that none of them could be used in isolation. We aim to follow-up and use PDF URLs provided by Unpaywall in large scale to control the ratio of publications which can be accessed.

\section{Conclusions}

It is clear that publishing as OA is on the rise in recent years. This trend is observed similarly in WOS and Scopus (while Scopus has higher raw publication counts but trends are identical) and based on OA identification stemming from both Unpaywall and Crossref. But still the majority of publications are closed access. We observed that despite the high coverage of Unpaywall (higher than $50 \%$ of articles and reviews in both WOS and Scopus), it doesn't provide enough metadata (as of April 2018) for OA categorization thus could be limiting for large scale OA monitoring in the leading bibliometric databases. Licence information from Crossref is more detailed and it gives a good possibility to complement Unpaywall metadata. Although we overcame the downsides by complementing these databases, we still found further contradictions between them with manual random checks. Some publications were OA (based on their licences or Unpaywall status) 
Table 4: OA status comparison between Unpaywall and Crossref in Scopus publications

\begin{tabular}{l|l|r|r}
\hline Crossref OA Status & Unpaywall OA Status & Frequency & Percent \\
\hline Closed Access & Closed Access & $5,138,444$ & 36.21 \\
\hline NA & Closed Access & $4,635,801$ & 32.67 \\
\hline NA & Open Access & $2,201,936$ & 15.52 \\
\hline Closed Access & Open Access & $1,549,902$ & 10.92 \\
\hline Open Access & Open Access & 502,510 & 3.54 \\
\hline Open Access & Closed Access & 160,132 & 1.13 \\
\hline NA & NA & 15 & 0.00 \\
\hline Open Access & NA & 4 & 0.00 \\
\hline Closed Access & NA & 1 & 0.00 \\
\hline
\end{tabular}

Table 5: Random sample OA status check on publications from WOS

\begin{tabular}{l|l|l|r|r}
\hline PDF Manually accessible? & Licence status & Pub OA? & Frequency & Percent \\
\hline PDF Accessible & Open Access & Unpaywall OA & 104 & 46.85 \\
\hline No Access to PDF & Closed Access & Unpaywall non-OA & 44 & 19.82 \\
\hline No Access to PDF & Open Access & Unpaywall non-OA & 18 & 8.11 \\
\hline No Access to PDF & Closed Access & Unpaywall OA & 16 & 7.21 \\
\hline PDF Accessible & Closed Access & Unpaywall OA & 16 & 7.21 \\
\hline PDF Accessible & Closed Access & Unpaywall non-OA & 14 & 6.31 \\
\hline No Access to PDF & Open Access & Unpaywall OA & 5 & 2.25 \\
\hline NA & Closed Access & Unpaywall non-OA & 1 & 0.45 \\
\hline No Access to PDF & Closed Access & Missing on Unpaywall & 1 & 0.45 \\
\hline PDF Accessible & NA & Unpaywall non-OA & 1 & 0.45 \\
\hline PDF Accessible & Open Access & Unpaywall non-OA & 1 & 0.45 \\
\hline PDF Accessible & NA & Unpaywall OA & & 0.45 \\
\hline
\end{tabular}

while their PDF files were not accessible through publishers' websites. Some publications were closed access, while their PDF files were accessible. We found that the issue of multiple records for some publications or multiple licence information is something that needs to be seriously considered in OA monitoring. While we tried to test different scenarios in OA identification, still there are publications that won't fit into any of the scenarios and we had to categorize them as $N A$ (since we wanted to keep the Closed Access definition as strict as possible), these are the publications that need to be further studied and usually the metadata of the OA databases are lacking for them. We propose OA monitoring activities to try to benefit from our approach in compelemting the metadata from OA databases, i.e. Unpaywall and Crossref, while noting that there are contradictions between these sources. Our effort to complement these databases proves that none of them could be used in isolation.

\section{References}

Archambault, E., Amyot, D., Deschamps, P., Nicol, A., Rebout, L., \& Roberge, G. (2013). Peer-reviewed papers at the european and world levels-2004-2011. Info@ Science, 1, 495-6505.

Crossref. (2018, October). Crossref. Retrieved from https://www.crossref.org/

CrossRef-API. (2019, October). CrossRef api. Retrieved from https://github.com/CrossRef/rest-api-doc\# filter-names

Jahn. (2017, January). About the hybrid oa dashboard. Retrieved from https://subugoe.github.io/hybrid_ oa_dashboard/about.html

Piwowar, H., Priem, J., Larivière, V., Alperin, J. P., Matthias, L., Norlander, B., .. Haustein, S. (2018). The state of oa: A large-scale analysis of the prevalence and impact of open access articles. PeerJ, 6, e4375.

Rimmert, C., Bruns, A., Lenke, C., \& Taubert, N. C. (2017). ISSN-matching of gold oa journals (issn-gold-oa) 2.0 .

Wohlgemuth, M., Rimmert, C., \& Winterhager, M. (2016). ISSN-matching of gold oa journals (issn-gold-oa). 
Table 6: Random sample OA status check on publications from Scopus

\begin{tabular}{l|l|l|r|r}
\hline PDF Manually accessible? & Licence status & Pub OA? & Frequency & Percent \\
\hline PDF Accessible & Open Access & Unpaywall OA & 105 & 46.26 \\
\hline No Access to PDF & Closed Access & Unpaywall non-OA & 48 & 21.15 \\
\hline No Access to PDF & Closed Access & Unpaywall OA & 17 & 7.49 \\
\hline PDF Accessible & Closed Access & Unpaywall OA & 17 & 7.49 \\
\hline No Access to PDF & Open Access & Unpaywall non-OA & 17 & 7.49 \\
\hline PDF Accessible & Closed Access & Unpaywall non-OA & 14 & 6.17 \\
\hline No Access to PDF & Open Access & Unpaywall OA & 4 & 1.76 \\
\hline NA & Closed Access & Unpaywall non-OA & 1 & 0.44 \\
\hline PDF Accessible & NA & Unpaywall non-OA & 1 & 0.44 \\
\hline PDF Accessible & Open Access & Unpaywall non-OA & 1 & 0.44 \\
\hline PDF Accessible & NA & Missing on Unpaywall & 1 & 0.44 \\
\hline PDF Accessible & NA & Unpaywall OA & 1 & 0.44 \\
\hline
\end{tabular}

